\title{
Intranasal Delivery of Exendin-4 Confers Neuroprotective Effect Against Cerebral Ischemia in Mice
}

\author{
Huinan Zhang, ${ }^{1}$ Jingru Meng, ${ }_{1}^{1}$ Shimeng Zhou, ${ }^{1}$ Yunhan Liu, ${ }^{2}$ Di Qu, ${ }^{1}$ Ling Wang, ${ }^{3}$ Xubo Li, ${ }^{1}$ Ning Wang, \\ Xiaoxing Luo, ${ }^{1,4}$ and Xue Ma ${ }^{1,4}$
}

Received 8 August 2015; accepted 4 December 2015; published online 21 December 2015

\begin{abstract}
Exendin-4 is now considered as a promising drug for the treatment of cerebral ischemia. To determine the neuroprotective effects of intranasal exendin-4, C57BL/6J mice were intranasally administered with exendin- 4 daily for 7 days before middle cerebral artery occlusion (MCAO) surgery. Intranasally administered exendin-4 produced higher brain concentrations and lower plasma concentrations when compared to identical doses administered interperitoneally. Neurological deficits and volume of infarcted lesions were analyzed $24 \mathrm{~h}$ after ischemia. Intranasal administration of exendin- 4 exhibited significant neuroprotection in C57BL/6 mice subjected to MCAO by reducing neurological deficit scores and infarct volume. The neuroprotective effects of exendin-4 were blocked by the knockdown of GLP$1 \mathrm{R}$ with shRNA. However, exendin-4 has no impact on glucose and insulin levels which indicated that the neuroprotective effect was mediated by the activation of GLP-1R in the brain. Exendin-4 intranasal administration restored the balance between pro- and anti-apoptotic proteins and decreased the expression of Caspase-3. The anti-apoptotic effect was mediated by the cAMP/PKA and PI3K/Akt pathway. These findings provided evidence that exendin-4 intranasal administration exerted a neuroprotective effect mediated by an anti-apoptotic mechanism in MCAO mice and protected neurons against ischemic injury through the GLP-1R pathway in the brain. Intranasal delivery of exendin-4 might be a promising strategy for the treatment of ischemic stroke.
\end{abstract}

KEY WORDS: cerebral ischemia; exendin-4; intranasal delivery; neuroprotection.

\section{INTRODUCTION}

Stroke is the second leading cause of death worldwide in people aged 60 years or older (1). In addition, stroke is a leading cause of long-term severe disability (2). About $87 \%$ of all strokes are represented by ischemic strokes, when the blood flow to the brain is blocked (2). So far, the only drug used for the treatment of acute ischemic stroke approved by the US Food and Drug Administration (FDA) is tissue plasminogen activator (tPA) (3), which is intravenously administered to dissolve blood clots and restore blood flow. However, this thrombolytic therapy has a limited time

Huinan Zhang, Jingru Meng and Shimeng Zhou contributed equally to this work.

$\overline{\text { Electronic supplementary material The online version of this article }}$ (doi:10.1208/s12248-015-9854-1) contains supplementary material, which is available to authorized users.

${ }^{1}$ Department of Pharmacology, School of Pharmacy, Fourth Military Medical University, 169 Changle West Rd., Xi'an, 710032, China.

${ }^{2}$ School of Nursing, The Fourth Military Medical University, Xi'an, China.

${ }^{3}$ Department of Health Statistics, Faculty of Preventative Medicine, The Fourth Military Medical University, Xi'an, China.

${ }^{4}$ To whom correspondence should be addressed. (e-mail: zhanghuinanshen@126.com; maxuel@fmmu.edu.cn) window for treatment, and it is associated with risks of intracerebral hemorrhage particularly in patients having large-volume lesions $(4,5)$.

Glucagon-like peptide-1 (GLP-1) is a gastrointestinal hormone which plays important roles in blood glucose control and is also considered as a neuropeptide synthesized by neuronal cells. Glucagon-like peptide-1 receptor (GLP-1R) was found to be widely expressed in the brain (6), and its activation protects against neurological disease (7). Exendin-4 is a long-lasting GLP-1R agonist which is widely used for the treatment of type 2 diabetes. The prolonged duration of its action and high apparent in vivo potency make exendin-4 more suitable as a potential pharmacological candidate. Besides its anti-diabetic effects, previous studies have shown that exendin-4 mediated neuroprotection in animal models of stroke (8-13). Intracerebroventricular injection of exendin-4 was found to exert neuroprotective effect against ischemic stroke in rats with middle cerebral artery occlusion (MCAO) surgery (8). Repeated administration of exendin-4 for 7 days was reported to reduce the infarct volume caused by MCAO in rats (10). Intravenous administration of exendin-4 provided neuroprotection against ischemic injury in mice at $1 \mathrm{~h}$ after MCAO, but the effect was lost at $3 \mathrm{~h}$ after MCAO (12). Moreover, in both young healthy and aged diabetic/obese mice, exendin-4 showed a neuroprotection against stroke induced by MCAO (9). Therefore, exendin- 4 is considered to 
be a promising strategy for the treatment of cerebral ischemia. However, the blood-brain barrier blocks most small molecules and nearly all large molecules from reaching the diseased brain; thus, systemic delivery of therapeutic peptides are often found to be ineffective $(14,15)$. Peptidic drugs need to be administered at large doses to achieve therapeutic levels in the brain, which would increase the systemic adverse effects. Although peptidic drugs can be directly injected into the brain via intracerebroventricular administration, this invasive technique is not suitable for clinical use $(8,16,17)$. Hence, a noninvasive approach to bypass the blood-brain barrier to target the brain should be worthwhile.

Intranasal administration is more convenient to the patient, and it alleviates the pain and discomfort associated with injections (18). In addition, this route has the potential to overcome the blood-brain barrier and reduce the side effects caused by systemic injection. Thus, this delivery system represents a novel alternative for the treatment of neurologic diseases $(19,20)$. Moreover, intranasal administration has the advantage of avoiding the gastrointestinal and hepatic metabolism $(21,22)$. The nasal cavity has a large surface area and nasal mucosa is highly vascularized; thus, the concentrations of neuroprotective peptides in the brain after intranasal administration are often higher than those observed by systemic injection (23). Furthermore, intranasal administration facilitates self-medication, thereby improving patient compliance compared with injections. Therefore, the intranasal route has aroused increasing interest as a route of administration for peptides. In the present study, we investigated the protective effects of intranasal administration of exendin- 4 on cerebral ischemia in MCAO mice and characterized the therapeutic potential of exendin- 4 through intranasal delivery for the treatment of cerebral ischemia.

\section{MATERIALS AND METHODS}

\section{Experimental Animals}

Male C57BL/6 mice from the Experimental Animal Center of Fourth Military Medical University weighing between 18 and $22 \mathrm{~g}$ were used in our study. All protocols were approved by the Animal Care and Use Committee of the Fourth Military Medical University. Every effort was made to minimize the number of animals used and their discomfort. Mice were randomly divided into the following seven groups ( $n=12$ for each group): group 1, sham group; group 2, intraperitoneally administered (i.p.) vehicle; group 3 , $0.5 \mu \mathrm{g} / \mathrm{kg}$ exendin-4 i.p.; group 4, $5.0 \mu \mathrm{g} / \mathrm{kg}$ exendin-4 i.p.; group 5, intranasal (i.n.) administration of vehicle; group 6 , $0.5 \mu \mathrm{g} / \mathrm{kg}$ exendin-4 i.n.; group $6,5.0 \mu \mathrm{g} / \mathrm{kg}$ exendin-4 i.n.. The drug was administered once a day for seven consecutive days. After the last administration, mice in groups 2-6 were subjected to MCAO surgery. Neurological deficits and volume of infarcted lesions were analyzed $24 \mathrm{~h}$ after ischemia.

\section{Intranasal Administration}

Mice were anesthetized by $1 \%$ pentobarbital sodium (10 $\mathrm{mg} / \mathrm{kg}$ intraperitoneal). After placing them on their backs, a total volume of $20 \mu \mathrm{l}$ exendin-4 or vehicle per mouse was administered through the nasal cavity. Alternating drops
(1 $\mu \mathrm{l} / \mathrm{drop}$ ) were administered between the left and right nares at the rate of 2 min per drop. To ensure that the drops were naturally inhaled into the nasal cavity, the opposite naris and mouth of the mice were kept closed during the administration.

\section{Determination of Blood Glucose and Plasma Insulin Levels}

Blood samples were collected at 0 and $30 \mathrm{~min}$ after intranasal administration of exendin-4. The plasma insulin level was determined using an ELISA kit (Millipore, Billerica, MA, USA). The blood glucose level was determined using a glucometer (Roche Diagnostics, Mannheim, Germany).

\section{Determination of Exendin-4 Levels in the Brain and Plasma}

C57BL/6 mice were intraperitoneally or intranasally treated with $0.5,5.0 \mu \mathrm{g} / \mathrm{kg}$ exendin- 4 , or vehicle. Six mice from each group were sacrificed at each time point $(0,0.25$, $0.5,1.0,1.5$, and $2.0 \mathrm{~h}$, Fig. 1a) after administration; then, brain tissue in the ischemic penumbra and plasma sample were collected from every animal. Exendin-4 levels in the brain and plasma were evaluated by ELISA (Phoenix Pharmaceuticals, Burlingame, CA, USA).

\section{The MCAO Model}

The MCAO model was performed according to a previously published protocol (24). Briefly, mice were anesthetized with $1 \%$ pentobarbital sodium. After the right common carotid artery (CCA) and the external carotid artery (ECA) were isolated, a nylon filament coated with silicon resin was gently advanced approximately $11 \mathrm{~mm}$ from the incision near the ECA-CCA branch. Then, the middle cerebral artery (MCA) was occluded. Reperfusion process was performed at $90 \mathrm{~min}$ after ischemia. The sham group underwent similar procedure, but the filament was just advanced for $5 \mathrm{~mm}$. The animals were placed on a heating pad on a surgical table soon after the anesthesia. During the entire surgical procedure, the body temperature was continuously monitored with a rectal probe and maintained at 37.0 $\pm 0.5^{\circ} \mathrm{C}$. Mice were kept in heated cages for the next $2 \mathrm{~h}$ ischemia, and rectal temperature was frequently measured. Next, the animals were placed in their home cages and allowed free access to food and water.

\section{Evaluation of Neurological Deficits}

Neurological deficit scores were analyzed according to Bederson's score $24 \mathrm{~h}$ after MCAO injury, which were defined as follows. No deficit observed corresponded to 0 point; flexion of the contralateral torso and forelimb corresponded to 1 point; decreased resistance to lateral push without circling corresponded to 2 points; leaning towards the affected side corresponded to 3 points; no spontaneous locomotor activity corresponded to 4 points. Higher neurological deficit scores indicated more severe impairment of motor injury. 


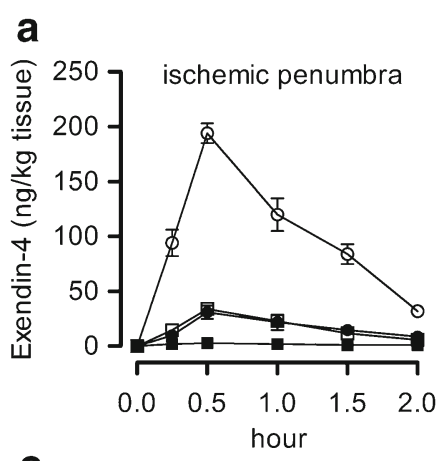

C

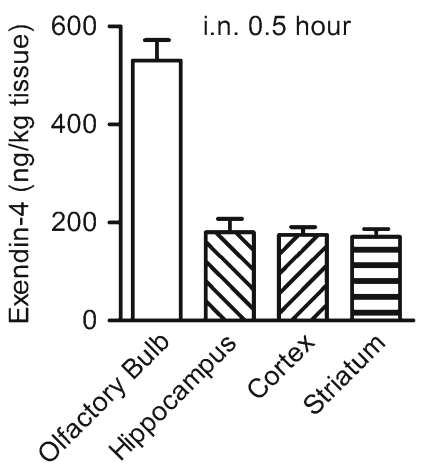

b

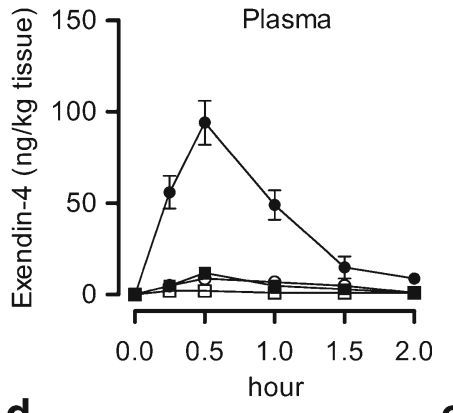

d

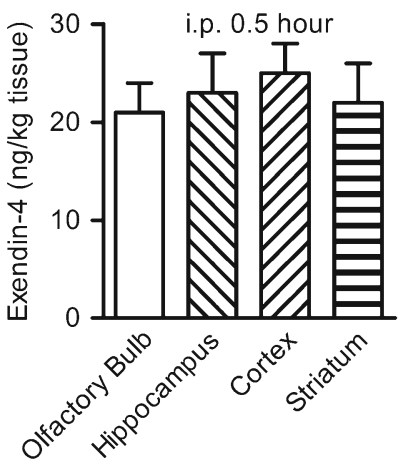

$\square 0.5 \mu \mathrm{g} / \mathrm{kg}$ (i.n.)

$\multimap 5.0 \mu \mathrm{g} / \mathrm{kg}($ i.n. $)$

- $0.5 \mu \mathrm{g} / \mathrm{kg}$ (i.p.)

$\rightarrow 5.0 \mu \mathrm{g} / \mathrm{kg}$ (i.p.)

e

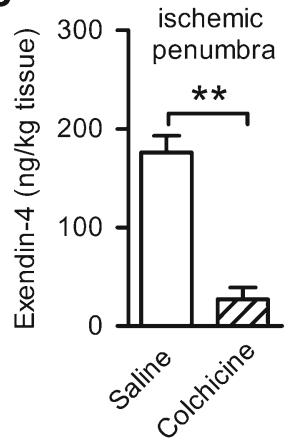

Fig 1. Exendin-4 entered into the brain after intranasal administration. a The concentration of exendin- 4 in the ischemic penumbra area after a single intraperitoneal or intranasal injection of 0.5 or $5.0 \mu \mathrm{g} / \mathrm{kg}$ exendin- $4, n=6$. b The concentration of exendin- 4 in the plasma after a single intraperitoneal or intranasal injection of 0.5 or $5.0 \mu \mathrm{g} / \mathrm{kg}$ exendin- $4, n=6$. c The concentration of exendin- 4 in different brain regions 30 min after a single intranasal injection of $5.0 \mu \mathrm{g} / \mathrm{kg}$ exendin- 4 . d The concentration of exendin-4 in different brain regions $30 \mathrm{~min}$ after a single intraperitoneal injection of $5.0 \mu \mathrm{g} / \mathrm{kg}$ exendin-4. e Effect of colchicine on transportation of intranasal exendin-4, saline, or colchicine was intranasal administrated $15 \mathrm{~min}$ before intranasal injection of $5.0 \mu \mathrm{g} / \mathrm{kg}$ exendin-4, $n=6$, i.n. intranasal, i.p. intraperitoneal, $* * p<0.01$

\section{Calculation of Infarcted Volume by 2-, 3-, 5-Triphenyltetrazolium Chloride (TTC) Staining}

Infarcted volume was analyzed by TTC staining after the evaluation of neurological deficit scores (25). The whole brain was removed and placed at $-20^{\circ} \mathrm{C}$ for $30 \mathrm{~min}$. Two-mm slices were cut and incubated in $1.5 \%$ TTC solution for $30 \mathrm{~min}$ at $37^{\circ} \mathrm{C}$ in the dark. Next, the slices were fixed in $4 \%$ paraformaldehyde for $24 \mathrm{~h}$ and digitally scanned. Infarct volume was calculated as the volume of the contralateral hemisphere minus the non-infarcted volume of the ipsilateral hemisphere using PhotoShop CS4 software and shown as the percentage of volume of the contralateral hemisphere.

\section{Knockdown of GLP-1R by Intracerebroventricular Infusion of shRNA}

The specific shRNA sequence targeting to the GLP-1R mRNA (GenBank accession no. NM_021332.2, nucleotides 608628) was constructed into the pGV118 lentiviral vector (GeneChem Co., Ltd., Shanghai, China). High purity ( $>1.9$ ratio 260/280 Abs) transfection ready vector DNA was prepared and resuspended in TE ( $\mathrm{pH}$ 8.0). The shRNA target sequence was GCCCTCAAGTGGATGTATAGC $\left(5^{\prime}\right.$ to $\left.3^{\prime}\right)$. A scrambled shRNA sequence TTCTCCGAACGTGTCACGT (5' to $\left.3^{\prime}\right)$ was chosen as a negative control. The lentivirus was intracerebroventricularly injected in the mice brain 7 days before intranasal administration of exendin-4. After anesthesia with $1 \%$ pentobarbital sodium, mice were placed in a stereotaxic frame with a mouse head holder. Lentivirus was stereotaxically injected into the right lateral ventricle at the coordinate of bregma -0.4 $\mathrm{mm}$, dorsoventral $0.7 \mathrm{~mm}$, lateral $1.8 \mathrm{~mm}$ via a burr hole. The needle was kept in the ventricle for $5 \mathrm{~min}$, and then, it was withdrawn at the rate of $1 \mathrm{~mm}$ per minute. After the bone wound was closed with bone wax, the mice were placed back into their cages after their recovery from anesthesia.

\section{Nissl Staining}

After neurological evaluation, the brains were removed and perfused with cold $4 \%$ paraformaldehyde. Brain blocks containing hippocampus and prefrontal cortex were cut into frozen section. Coronal sections at the thickness of $10 \mu \mathrm{m}$ were cut using a Leica CM1800 Cryostat (Leica Microsystems) and then stained with $0.1 \%$ cresyl violet for $20 \mathrm{~min}$. The sections were evaluated using light microscopy (Olympus BX60).

\section{Western Blot}

Brain sample was harvested from the ischemic penumbra region on the operated side at $24 \mathrm{~h}$ after reperfusion. An 
equal amount of protein $(10 \mu \mathrm{g})$ was loaded into each lane and subjected to sodium dodecyl sulfate-polyacrylamide gel electrophoresis (SDS-PAGE) at $200 \mathrm{~V}$ for $45 \mathrm{~min}$. Protein bands were transferred into polyvinyl difluoride membranes at $100 \mathrm{~V}$ for $2 \mathrm{~h}$. Membranes were incubated overnight with a rabbit anti-p-AKT antibody (1:1000; Santa Cruz Biotechnology, Santa Cruz, CA, USA), p-CREB (1:1000; Santa Cruz), GLP-1R (1:1000; Santa Cruz), and $\beta$-actin (1:5000; Sigma) at $4^{\circ} \mathrm{C}$ followed by goat anti-rabbit $\mathrm{IgG}$ secondary antibody (1:10000; Santa Cruz) for $1 \mathrm{~h}$. Then, the bands on the film were scanned and analyzed.

\section{Statistical Analysis}

Statistical analysis was performed using SPSS 13.0. All data, except for neurological scores, were expressed as mean $\pm \mathrm{SD}$, and differences between groups were analyzed by one-way ANOVA followed by Dunnett $T$ test. The neurological scores were analyzed using the KruskalWallis test followed by the Mann-Whitney $U$ test with the Bonferroni correction. A value of $p<0.05$ was considered statistically significant.

\section{RESULTS}

\section{Exendin-4 Accumulated in the Brain After Intranasal Administration}

Exendin-4 was intranasally or intraperitoneally administered at the dose of 0.5 or $5.0 \mu \mathrm{g} / \mathrm{kg}$ in C $57 \mathrm{BL} / 6$ mice. The concentration of exendin-4 was detected in the ischemic penumbra area and plasma at different time points after injection. At $15 \mathrm{~min}, 0.5 \mathrm{~h}, 1 \mathrm{~h}, 1.5 \mathrm{~h}$, and $2 \mathrm{~h}$ after administration, the concentration of exendin- 4 in the ischemic penumbra area after its intranasal administration at a dose of $0.5 \mu \mathrm{g} / \mathrm{kg}$ was equivalent to the concentration reached after its intraperitoneal administration at a dose of $5 \mu \mathrm{g} / \mathrm{kg}$. Exendin-4 reached the peak level in the ischemic penumbra area $30 \mathrm{~min}$ after intranasal administration, and then, it slowly decreased (Fig. 1a). Brain exendin-4 was still detectable $2 \mathrm{~h}$ after i.n. administration of $5.0 \mu \mathrm{g} / \mathrm{kg}$. In contrast, the plasma level was barely detectable after two i.n. doses of exendin-4 (Fig. 1b). The uptake and distribution of exendin- 4 by different brain regions after intranasal or intraperitoneal injection was further explored. The concentration of exendin-4 into the olfactory bulb after intranasal administration was significantly higher than other brain tissue (Fig. 1c), while no significant difference in exendin-4 level was found in the whole brain after intraperitoneal injection (Fig. 1d). To further analyze the transport mechanism after intranasal administration, colchicine, a microtubule assembly inhibitor which can reduce axonal transport (26), was intranasally administered $15 \mathrm{~min}$ before intranasal administration of exendin-4. A very low concentration of exendin- 4 was detected in the ischemic penumbra area after administration of colchicine (Fig. 1e). These data showed a rapid accumulation of exendin- 4 in the brain after intranasal administration and demonstrated that the anterograde axonal transport is essential for this delivery process.

\section{Intranasal Administration of Exendin-4 Improved Functional Outcome and Reduced Infarction Size Caused by MCAO}

The neuroprotective effects of exendin-4 administered intranasally was evaluated in MCAO mice, which mimics the most common type of stroke in humans. Eleven mice (3 in $\mathrm{MCAO}+$ vehicle i.p., MCAO + vehicle i.n. and MCAO + 0.5 $\mu \mathrm{g} / \mathrm{kg}$ exendin-4 i.p. group, and 1 in $\mathrm{MCAO}+5.0 \mu \mathrm{g} / \mathrm{kg}$ exendin-4 i.p. and $\mathrm{MCAO}+0.5 \mu \mathrm{g} / \mathrm{kg}$ exendin- 4 i.n. group) were excluded because of death after surgery. Twenty-four hours after MCAO injury, remarkably higher neurological deficit scores were measured and an obvious area of infarction spanned the right frontal, parietal, and occipital cerebral cortices compared to the sham group (Fig. 2a-c). The neurological deficit score and infarct volume caused by MCAO was not significantly reduced by intraperitoneal administration of exendin- 4 at $0.5 \mu \mathrm{g} / \mathrm{kg}$. However, intranasal administration of exendin-4 at $0.5 \mu \mathrm{g} / \mathrm{kg}$ exerted neuroprotective effect by reducing neurological deficit scores and infarct volume. Intranasal administration of exendin-4 at 5.0 $\mu \mathrm{g} / \mathrm{kg}$ further improved the functional outcome and reduced the infarction size caused by MCAO (Fig. 2a-c).

\section{Neuroprotective Effect of Intranasal Administration of Exendin-4 Was Independent of the Periphery Effect}

The GLP-1R is widely expressed throughout the body, and its beneficial effect can be induced by direct central or indirect peripheral effect. Hyperglycemia is often associated with increased oxidative stress and inflammation; thus, it represents a major risk factor for cerebral ischemia $(27,28)$. The anti-stroke efficacy of exendin-4 in diabetic rats was reported by stimulating insulin release and hypoglycemic activity $(13,29)$. Since exendin-4 was detected in plasma after intranasal administration, the peripheral effect of exendin-4 needs to be excluded. In this study, intranasal administration of exendin-4 has no effect on blood glucose and plasma insulin level in non-diabetic mice (Fig. 3a, b). Thus, these results indicated that the neuroprotective effect of exendin-4 intranasal administration was not dependent on its peripheral effect against hyperglycemia by stimulating insulin secretion.

\section{Intranasal Administration of Exendin-4 Exerted Neuropro- tective Effect Against Cerebral Ischemia by Activating the GLP-1R in the Brain}

To further determine the mechanisms of neuroprotective effect, the shRNA lentiviral vectors were used to suppress GLP-1R expression in the ischemic penumbra area by stereotaxic injection of shRNA coated by lentivirus. A parallel analysis was performed using the negative control shRNA. Seven days after stereotaxis injection of shRNA, no significant difference in the GLP-1R level between control group and negative shRNA group was observed. However, the GLP-1R expression was significantly reduced to $30 \%$ of its original expression in the ischemic penumbra area 7 days after the unilateral administration of the shRNA vector targeting GLP-1R at the titer of $10^{8} \mathrm{TU} / \mathrm{ml}$ (Fig. $4 \mathrm{a}, \mathrm{b}$ ). Lentiviral coating shRNA against GLP-1R at the titer of $10^{6}$ and $10^{7}$ failed to silence the GLP-1R (Supplementary Figure 1A, B). This effect lasted at least for 21 days after 

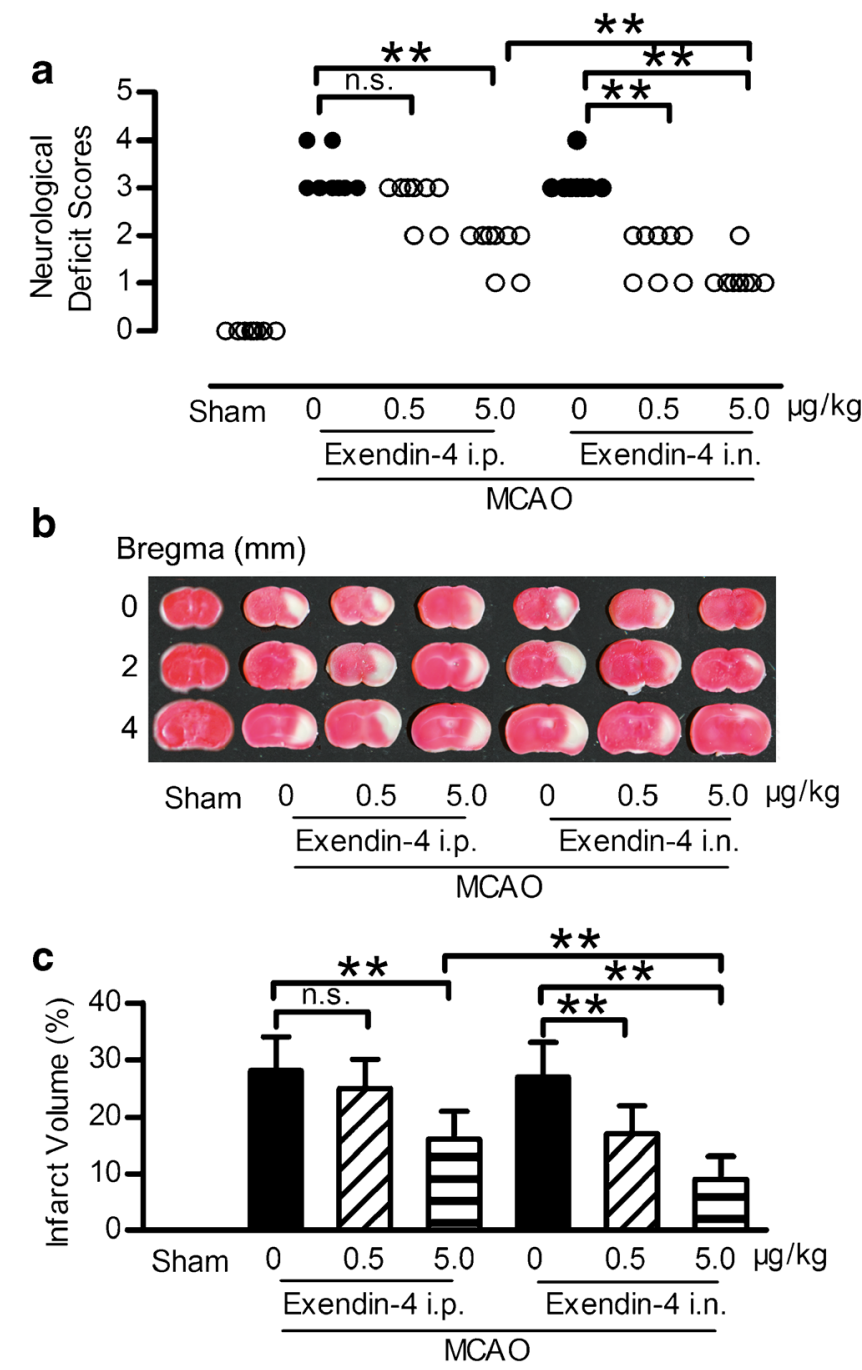

Fig 2. Intranasal administration of exendin-4 improved the neurological deficit and reduced infarction volume in focal brain ischemia mice. a Representative neurological deficit score $24 \mathrm{~h}$ after ischemia and reperfusion, $n=8$. b Brain slices stained with TTC $24 \mathrm{~h}$ after ischemia and reperfusion. $\mathbf{c}$ Quantitative analysis of $\mathbf{b}, n=6$, i.n. intranasal, i.p. intraperitoneal, $* * p<0.01$

injection (Supplementary Figure 1C, D). Seven days after the administration of the shRNA, exendin- 4 or vehicle was intranasally administered for 7 days and then subjected to MCAO. Neurological deficit score was evaluated and TTC
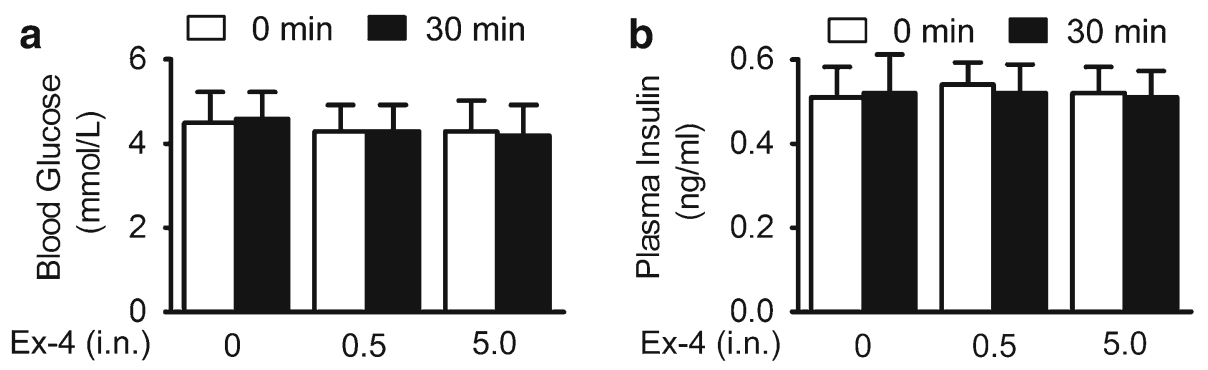

Fig. 3. Intranasal administration of exendin- 4 had no impact on blood glucose and plasma insulin level in non-diabetic mice. a Blood glucose levels in overnight-fasted C57BL/6 mice performed $30 \mathrm{~min}$ after intranasal injection of saline or 0.5 or $5.0 \mu \mathrm{g} / \mathrm{kg}$ exendin- 4 . b Plasma insulin levels in overnight-fasted mice performed $30 \mathrm{~min}$ after intranasal injection of saline or 0.5 or $5.0 \mu \mathrm{g} / \mathrm{kg}$ exendin- $4, n=12$ 
a

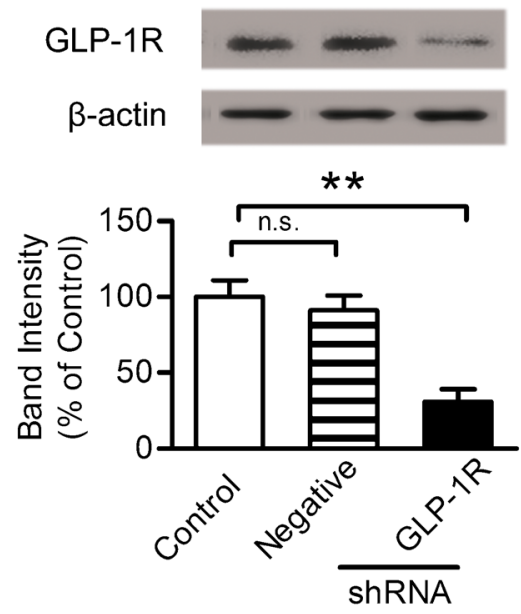

C

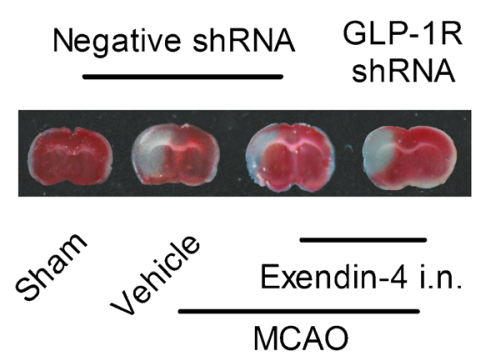

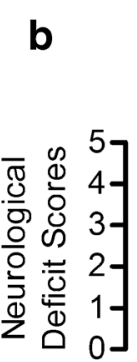

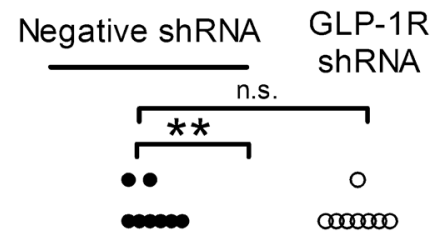

$\infty 0$

$0 \infty 0$

00000
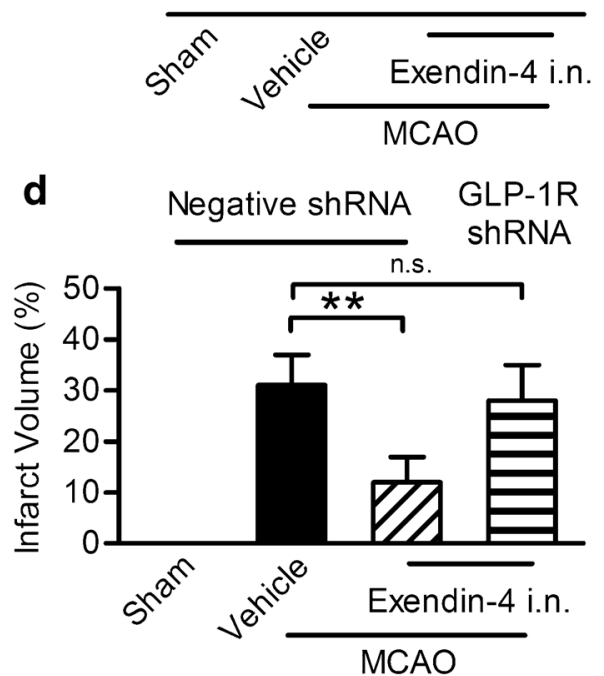

Fig. 4. Neuroprotective effect of exendin-4 intranasal administration blocked by intracerebroventricular infusion of GLP-1R shRNA in the ipsilateral ventricle. a Western blot analysis of GLP-1R expression. b Quantitative results of a. Results are representative of five independent experiments from five mice. c Representative neurological deficit score $24 \mathrm{~h}$ after ischemia and reperfusion, $n=8$. d Brain slices stained with TTC $24 \mathrm{~h}$ after ischemia and reperfusion and the quantitative analysis of infarct volume, $n=6$, i.n. intranasal, ${ }^{* *} p<0.01$

staining was performed $24 \mathrm{~h}$ after MCAO. Negative shRNA has no impact on the protective effect of intranasal exendin-4, while the GLP-1R shRNA almost totally abrogated its beneficial effect (Fig. 4c, d). These results indicated that the neuroprotective effect of intranasal exendin-4 relied upon activation of GLP-1R in the brain.

\section{Intranasal Administration of Exendin-4 Activated GLP-1R Signaling Pathway}

The downstream activation of GLP-1R leads to the stimulation of multiple signaling pathways (30). The activation of the signaling pathways depends on factors such as cell types, cellular stimuli, and environmental conditions. The cAMP/PKA/CREB and the PI3K/Akt pathway plays a very critical role in the process of cellular survival following neuronal injury $(31,32)$, MCAO injury led to the decrease of p-CREB and p-Akt level in the hippocampus, intranasal exendin- 4 reversed this injury, and GLP-1R shRNA blocked this trend (Fig. 5a-c). While in the olfactory bulb area, MCAO injury had no impact on the p-CREB and p-Akt level compared with the control group, intranasal administration of exendin- 4 increased the p-CREB and p-Akt level in the olfactory bulb area, and knockdown of GLP-1R by shRNA had no impact on this effect (Fig. 5d-f). These results indicated that intranasal exendin-4 exerted the stimulation of both the cAMP/PKA and PI3K/Akt pathways.

\section{Intranasal Administration of Exendin-4 Inhibited Neuronal Apoptosis Induced by MCAO}

The cAMP/PKA/CREB and PI3K/Akt pathways mediated the anti-apoptotic effect and reduced neurons death caused by MCAO. Therefore, the anti-apoptotic effect of intranasal exendin-4 against cerebral ischemia was evaluated by detecting the apoptotic related protein Caspase-3. Caspase- 3 is considered as a key mediator of apoptosis. MCAO injury led to the upregulation of Caspase-3 in the hippocampus, a phenomenon that was not observed in the sham group. This trend was reversed by the intranasal administration of exendin-4, while GLP-1R shRNA reduced this trend (Fig. 6a, b). The MCAO injury led to neuronal damage both in the prefrontal cortex and hippocampus CA1 region evaluated by Nissl staining. The injured neurons showed shrunken cell bodies accompanied by shrunken and pyknotic nuclei. Exendin-4 intranasal administration significantly decreased the number of injured neurons and preserved the original morphology of the neurons, which was modified by MCAO. However, this effect was blocked by GLP-1R shRNA (Fig. 6c-e). These results showed that 
a

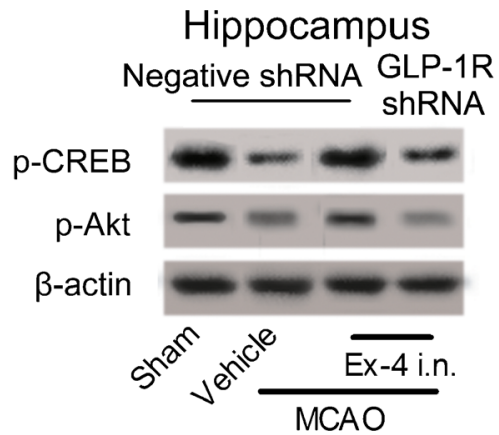

b

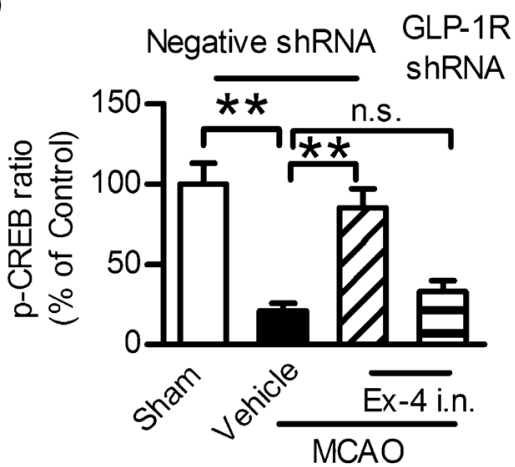

C

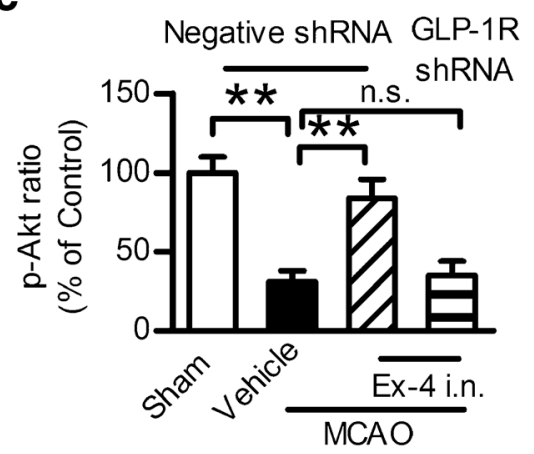

d

Olfactory Bulb
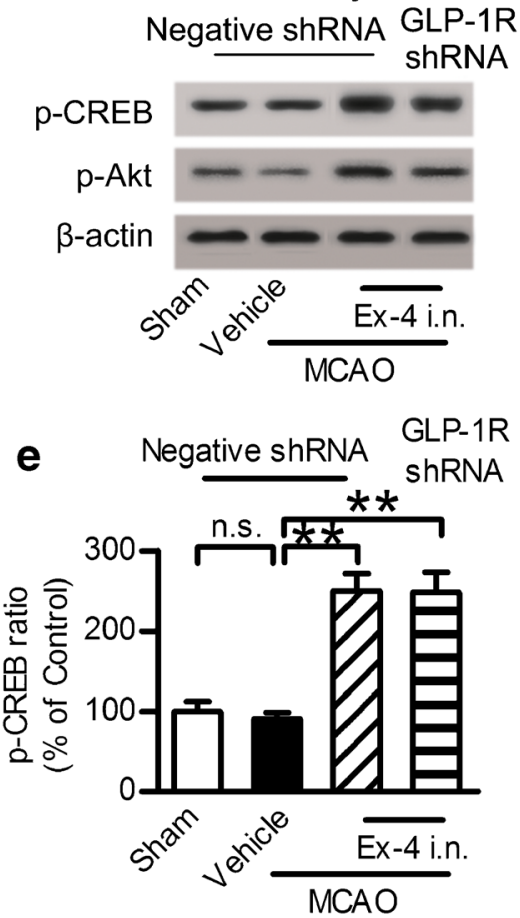

f

Negative shRNA GLP-1R

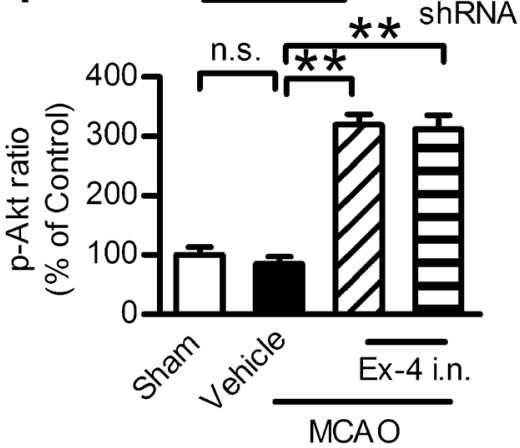

Fig. 5. Intranasal administration of exendin- 4 activated the GLP-1R signaling pathway. a Western blot analysis of p-CREB and p-Akt expression in the hippocampus. b, c Quantitative results of a. d Western blot analysis of p-CREB and p-Akt expression in the olfactory bulb. e, f Quantitative results of e. Results are representative of five independent experiments from five mice. Ex-4 exendin-4, i.n. intranasal, $* * p<0.01$

intranasal administration of exendin-4 exerted an antiapoptotic effect against neuronal injury induced by ischemic stroke.

\section{DISCUSSION}

Exendin- 4 is in clinical use for the treatment of diabetes and several studies indicate that it may be useful in the treatment of ischemic stroke (8-13), but there is no study to evaluate the effect of intranasal administration of exendin-4 on cerebral ischemia. We therefore conducted experiments to determine the neuroprotective effects of intranasal administration of exendin-4 on cerebral ischemia in MCAO mice.

Intranasal administration is a useful method of delivering therapeutic peptides to the brain for the treatment of central nervous system (CNS) diseases. After intranasal administration, the peptide uptake by the brain is 5-10 times higher than systemic administration (23). Moreover, peptides delivered by the intranasal route often have a longer permanence time in the brain than those delivered by systemic injection (33), which is in accordance with our research. Intranasal delivery is noninvasive and allows large molecules that do not cross the blood-brain barrier access to the brain. Therapeutic peptides are directly targeted to the CNS with intranasal administration, potentially reducing peripheral exposure and thus unwanted systemic side effects.

In our current study, intranasal administration of exendin- 4 at the dose of $0.5 \mu \mathrm{g} / \mathrm{kg}$ exerted neuroprotective effect by reducing neurological deficit scores and infarct volume. However, intraperitoneal administration with the 

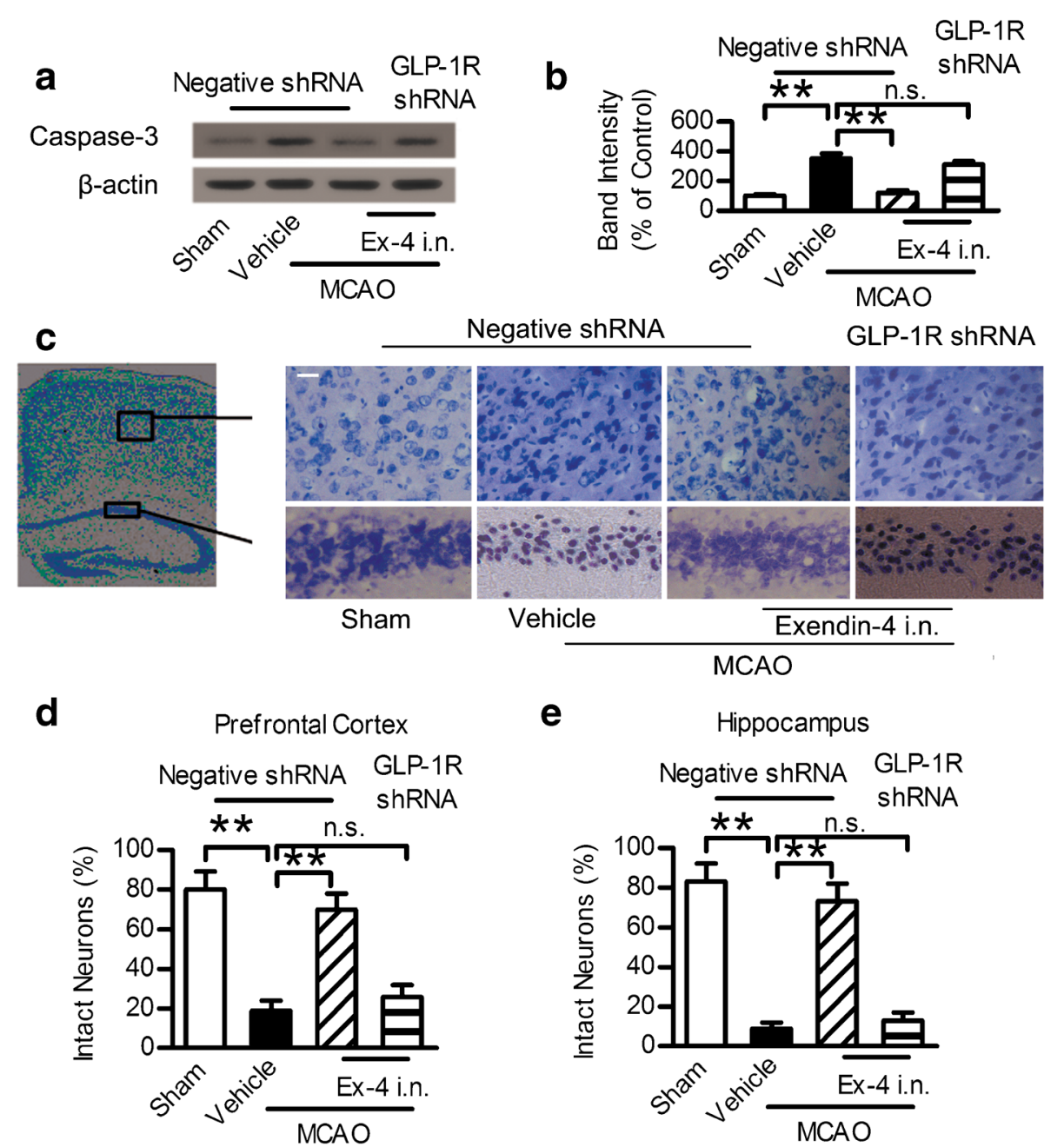

Fig. 6. Intranasal administration of exendin-4 exerted anti-apoptotic effect against focal brain ischemia. a Western blot analysis of Caspase-3 expression in ischemic penumbra area. b Quantitative results of a. c Photographs of Nissl staining in the hippocampus CA1 region and prefrontal cortex. Scale bar $=20 \mu \mathrm{m}$. d, e Percentage of healthy neurons in the hippocampus CA1 region and prefrontal cortex. Six different fields were counted per division in three separated experiments. Ex-4 exendin-4, i.n. intranasal, $* * p<0.01$

dose of $0.5 \mu \mathrm{g} / \mathrm{kg}$ did not reduce ischemic damage. Although a study (9) demonstrated a significant neuroprotective effect of exendin-4 with lower dose $(0.2 \mu \mathrm{g} / \mathrm{kg})$, it has to be noted that mice received the first administration of exendin- 4 at the far higher dose of 5 or $50 \mu \mathrm{g} / \mathrm{kg}$ after MCAO, and then, the treatment continued for 7 days with lower dose of the drug $(0.2 \mu \mathrm{g} / \mathrm{kg})$. The results showed neuroprotective efficacy of $50 \mu \mathrm{g} / \mathrm{kg}$ exendin- 4 at 1.5 and $3 \mathrm{~h}$ after stroke, but the $5 \mu \mathrm{g} / \mathrm{kg}$ dose was neuroprotective at $1.5 \mathrm{~h}$ only. The most efficacious dose of exendin-4 in their study was a bolus of $50 \mu \mathrm{g} / \mathrm{kg}$ followed by continued injections of $0.2 \mu \mathrm{g} / \mathrm{kg}$. Decreasing the first bolus injection from 50 to $5 \mu \mathrm{g} / \mathrm{kg}$ resulted in diminished efficacy. Therefore, the discrepancy between our research and study by Darsalia et al. are likely due to the different methods of administration.

Previous studies indicated that intranasally administered therapeutics reach the brain via the two main direct pathways, the olfactory and the trigeminal neural pathways. Direct delivery of drugs from the nose to the brain is initially attributed to the olfactory pathway (34-36). After intranasal administration, peptidic agents usually accumulate in the olfactory bulbs of the brain at the highest concentration and are then distributed throughout the rest of the brain $(37,38)$. Therefore, the olfactory neural pathway is likely one of the largest contributors to intranasal drug delivery. Our data indicated that entry of exendin- 4 into the olfactory bulb after intranasal administration was significantly higher than other brain tissue and suggested the olfactory pathway seems to play a pronounced role.

There are two possible mechanisms for the molecule to reach the brain though the olfactory nerve pathways, the extracellular and the intracellular mechanisms. The extracellular transportation is a rapid process which is permitted to occur only few minutes within olfactory cells (39). In contrast, the intracellular transport mechanism is a slow process and lasts for over several hours by axonal transportation (40). Our data indicated that exendin-4 accumulated in the brain $30 \mathrm{~min}$ after intranasal 
administration, then decreased slowly and lasted for $2 \mathrm{~h}$ after administration. Previous study published values for fast $(5.4 \mathrm{~mm} / \mathrm{h})$ and slow $(1.5 \mathrm{~mm} / \mathrm{h})$ axonal transport of horseradish peroxidase in olfactory nerves and calculated it would take $0.74 \mathrm{~h}$ (fast) $-2.7 \mathrm{~h}$ (slow) for intracellular transport within olfactory neurons to the olfactory bulb (41). From the perspective of axonal transport, this range of speeds is compatible with both retrograde $(4-8 \mathrm{~mm} / \mathrm{h})$ and fast anterograde transport $(2.1-16.6 \mathrm{~mm} / \mathrm{h})(42,43)$. In our present study, the time for intranasal exendin- 4 to reach the brain was close to the time of the fast axonal transport in olfactory nerves. Because transportation relies on microtubules, depolymerization of those microtubules by colchicine caused some vesicles to target the basolateral surface. Colchicine was used in previous studies to inhibit microtubule assembly and reduces axonal transport (40,43-46). We next blocked the axonal transport of olfactory by colchicine to examine the possibility that exendin- 4 may be transported by an axonal transport system in olfactory neural pathways. Our data showed that administration of colchicine significantly decreased the brain concentration of exendin-4 to a low level. Such an effect is consistent with previous report by Guardia et al. that a very low concentration of aTf was detected in the olfactory bulb when colchicine was administered before the intranasal administration of aTf (40). Based on our results, we speculated that exendin-4 could be transported via fast anterograde axonal transport and intracellular transport mechanisms may play a critical part in this process.

Neuroprotective strategies aimed at decreasing brain damage after ischemic stroke have failed to be translated into the clinical setting along the past decades (47). Up to now, tPA is still the only approved pharmacological treatment for ischemic stroke. Although most of the current therapies are focused on posttreatment after cerebral ischemia, accumulating lines of evidence have demonstrated the efficacy of pretreatment therapies which could induce neuroprotection against cerebral ischemic injury. As for exendin-4, the data from several references have demonstrated that administration of exendin- 4 before stroke resulted in neuroprotection $(8,10,11,13)$. In our current study, mice received exendin-4 intranasally before MCAO injury and data showed that pretreatment with intranasal administration of exendin-4 exerted neuroprotective effect by reducing neurological deficit scores and infarct volume. These results indicated the potential use of exendin- 4 for the treatment of stroke in type 2 diabetes patients or individuals at high risk to suffer from a stroke (e.g., pretreatment strategies).

\section{CONCLUSIONS}

Exendin-4 has been applied in clinic for the treatment of type 2 diabetes mellitus. However, the neuroprotective effect of exendin-4 against cerebral ischemia is gaining recognition. Our animal research indicated that the intranasal administration may be a more effective way to use exendin-4 against cerebral ischemia. Obviously, the application of our findings to human will need to be examined.

\section{ACKNOWLEDGMENTS}

This work has been supported by three grants from the National Natural Science Foundation of China (81571278, 81471093, and 81201515).

\section{COMPLIANCE WITH ETHICAL STANDARDS}

Conflict of Interest The authors declare that they have no competing interests.

\section{REFERENCES}

1. Lozano R, Naghavi M, Foreman K, Lim S, Shibuya K, Aboyans $\mathrm{V}$, et al. Global and regional mortality from 235 causes of death for 20 age groups in 1990 and 2010: a systematic analysis for the Global Burden of Disease Study 2010. Lancet. 2012;380(9859):2095-128.

2. Mozaffarian D, Benjamin EJ, Go AS, Arnett DK, Blaha MJ, Cushman M, et al. Heart disease and stroke statistics-2015 update: a report from the American Heart Association. Circulation. 2015;131(4):e29-322.

3. Go AS, Mozaffarian D, Roger VL, Benjamin EJ, Berry JD, Blaha MJ, et al. Heart disease and stroke statistics-2014 update: a report from the American Heart Association. Circulation. 2014;129(3):e28-292.

4. Lansberg MG, Thijs VN, Bammer R, Kemp S, Wijman CA, Marks MP, et al. Risk factors of symptomatic intracerebral hemorrhage after tPA therapy for acute stroke. Stroke. 2007;38(8):2275-8.

5. Fonarow GC, Smith EE, Saver JL, Reeves MJ, Bhatt DL, GrauSepulveda MV, et al. Timeliness of tissue-type plasminogen activator therapy in acute ischemic stroke: patient characteristics, hospital factors, and outcomes associated with door-to-needle times within 60 minutes. Circulation. 2011;123(7):750-8.

6. Heppner KM, Kirigiti M, Secher A, Juel Paulsen S, Buckingham $\mathrm{R}$, Pyke $\mathrm{C}$ et al. Expression and distribution of glucagon-like peptide-1 receptor mRNA, protein and binding in the male nonhuman primate (Macaca mulatta) brain. Endocrinology 2014:en20141675.

7. Holscher C. Central effects of GLP-1: new opportunities for treatments of neurodegenerative diseases. J Endocrinol. 2014;221(1):T31-41.

8. Li Y, Perry T, Kindy MS, Harvey BK, Tweedie D, Holloway HW, et al. GLP-1 receptor stimulation preserves primary cortical and dopaminergic neurons in cellular and rodent models of stroke and Parkinsonism. Proc Natl Acad Sci U S A. 2009;106(4):128590 .

9. Darsalia V, Hua S, Larsson M, Mallard C, Nathanson D, Nystrom $\mathrm{T}$, et al. Exendin-4 reduces ischemic brain injury in normal and aged type 2 diabetic mice and promotes microglial M2 polarization. PLoS One. 2014;9(8):e103114.

10. Briyal S, Gulati K, Gulati A. Repeated administration of exendin-4 reduces focal cerebral ischemia-induced infarction in rats. Brain Res. 2012;1427:23-34.

11. Lee CH, Yan B, Yoo KY, Choi JH, Kwon SH, Her S, et al. Ischemia-induced changes in glucagon-like peptide-1 receptor and neuroprotective effect of its agonist, exendin-4, in experimental transient cerebral ischemia. J Neurosci Res. 2011;89(7):1103-13.

12. Teramoto S, Miyamoto N, Yatomi K, Tanaka Y, Oishi H, Arai H, et al. Exendin-4, a glucagon-like peptide-1 receptor agonist, provides neuroprotection in mice transient focal cerebral ischemia. J Cereb Blood Flow Metab. 2011;31(8):1696-705.

13. Darsalia V, Mansouri S, Ortsater H, Olverling A, Nozadze N, Kappe C, et al. Glucagon-like peptide-1 receptor activation reduces ischaemic brain damage following stroke in type 2 diabetic rats. Clin Sci. 2012;122(10):473-83. 
14. Yang D, Sun YY, Lin X, Baumann JM, Warnock M, Lawrence DA, et al. Taming neonatal hypoxic-ischemic brain injury by intranasal delivery of plasminogen activator inhibitor-1. Stroke. 2013;44(9):2623-7.

15. Yang D, Sun YY, Lin X, Baumann JM, Dunn RS, Lindquist DM, et al. Intranasal delivery of cell-penetrating anti-NF-kappaB peptides (Tat-NBD) alleviates infection-sensitized hypoxic-ischemic brain injury. Exp Neurol. 2013;247:447-55.

16. Shi QJ, Xiao L, Zhao B, Zhang XY, Wang XR, Xu DM, et al. Intracerebroventricular injection of HAMI 3379, a selective cysteinyl leukotriene receptor 2 antagonist, protects against acute brain injury after focal cerebral ischemia in rats. Brain Res. 2012;1484:57-67.

17. Zhou S, Yu G, Chi L, Zhu J, Zhang W, Zhang Y, et al. Neuroprotective effects of edaravone on cognitive deficit, oxidative stress and tau hyperphosphorylation induced by intracerebroventricular streptozotocin in rats. Neurotoxicology. 2013;38:136-45.

18. Fletcher L, Isgor E, Sprague S, Williams LH, Alajajian BB, Jimenez DF, et al. Spatial distribution of insulin-like growth factor binding protein-2 following hypoxic-ischemic injury. BMC Neurosci. 2013;14:158.

19. Hanson LR, Frey WH. Intranasal delivery bypasses the bloodbrain barrier to target therapeutic agents to the central nervous system and treat neurodegenerative disease. BMC Neurosci. 2008;9 Suppl 3:S5.

20. Liu XF, Fawcett JR, Thorne RG, DeFor TA, Frey 2nd WH. Intranasal administration of insulin-like growth factor-I bypasses the blood-brain barrier and protects against focal cerebral ischemic damage. J Neurol Sci. 2001;187(1-2):91-7.

21. Pontiroli AE. Peptide hormones: review of current and emerging uses by nasal delivery. Adv Drug Deliv Rev. 1998;29(1-2):81-7.

22. Chien YW, Chang SF. Intranasal drug delivery for systemic medications. Crit Rev Ther Drug Carrier Syst. 1987;4(2):67-194.

23. Nonaka N, Farr SA, Kageyama H, Shioda S, Banks WA Delivery of galanin-like peptide to the brain: targeting with intranasal delivery and cyclodextrins. J Pharmacol Exp Ther. 2008;325(2):513-9.

24. de la Rosa X, Cervera A, Kristoffersen AK, Valdes CP, Varma $\mathrm{HM}$, Justicia C, et al. Mannose-binding lectin promotes local microvascular thrombosis after transient brain ischemia in mice. Stroke. 2014;45(5):1453-9.

25. Dong YF, Wang LX, Huang X, Cao WJ, Lu M, Ding JH, et al. Kir6.1 knockdown aggravates cerebral ischemia/reperfusioninduced neural injury in mice. CNS Neurosci Ther. 2013;19(8):617-24.

26. Biswas K, Das SJ. Effect of microtubule disruption on neuronal spread and replication of demyelinating and nondemyelinating strains of mouse hepatitis virus in vitro. J Virol. 2014;88(5):30437.

27. Kamada H, Yu F, Nito C, Chan PH. Influence of hyperglycemia on oxidative stress and matrix metalloproteinase-9 activation after focal cerebral ischemia/reperfusion in rats: relation to blood-brain barrier dysfunction. Stroke. 2007;38(3):1044-9.

28. Sander D, Kearney MT. Reducing the risk of stroke in type 2 diabetes: pathophysiological and therapeutic perspectives. J Neurol. 2009;256(10):1603-19.

29. Drucker DJ, Nauck MA. The incretin system: glucagon-like peptide-1 receptor agonists and dipeptidyl peptidase-4 inhibitors in type 2 diabetes. Lancet. 2006;368(9548):1696-705.
30. Aronis KN, Chamberland JP, Mantzoros CS. GLP-1 promotes angiogenesis in human endothelial cells in a dose-dependent manner, through the Akt, Src and PKC pathways. Metab Clin Exp. 2013;62(9):1279-86.

31. Kitagawa K. CREB and cAMP response element-mediated gene expression in the ischemic brain. FEBS J. 2007;274(13):3210-7.

32. Simao F, Matte A, Pagnussat AS, Netto CA, Salbego CG. Resveratrol prevents CA1 neurons against ischemic injury by parallel modulation of both GSK-3beta and CREB through PI3K/Akt pathways. Eur J Neurosci. 2012;36(7):2899-905.

33. Dogrukol-Ak D, Banks WA, Tuncel N, Tuncel M. Passage of vasoactive intestinal peptide across the blood-brain barrier. Peptides. 2003;24(3):437-44.

34. Thorne RG, Emory CR, Ala TA, Frey 2nd WH. Quantitative analysis of the olfactory pathway for drug delivery to the brain. Brain Res. 1995;692(1-2):278-82.

35. Chen XQ, Fawcett JR, Rahman YE, Ala TA, Frey IW. Delivery of nerve growth factor to the brain via the olfactory pathway. J Alzheimers Dis : JAD. 1998;1(1):35-44.

36. Chow HS, Chen Z, Matsuura GT. Direct transport of cocaine from the nasal cavity to the brain following intranasal cocaine administration in rats. J Pharm Sci. 1999;88(8):754-8.

37. Thorne RG, Hanson LR, Ross TM, Tung D, Frey 2nd WH. Delivery of interferon-beta to the monkey nervous system following intranasal administration. Neuroscience. 2008;152(3):785-97.

38. Lochhead JJ, Thorne RG. Intranasal delivery of biologics to the central nervous system. Adv Drug Deliv Rev. 2012;64(7):614-28.

39. Pardeshi CV, Belgamwar VS. Direct nose to brain drug delivery via integrated nerve pathways bypassing the blood-brain barrier: an excellent platform for brain targeting. Expert Opin Drug Deliv. 2013;10(7):957-72.

40. Guardia Clausi M, Paez PM, Campagnoni AT, Pasquini LA, Pasquini JM. Intranasal administration of aTf protects and repairs the neonatal white matter after a cerebral hypoxicischemic event. Glia. 2012;60(10):1540-54.

41. Buchner K, Seitz-Tutter D, Schonitzer K, Weiss DG. A quantitative study of anterograde and retrograde axonal transport of exogenous proteins in olfactory nerve C-fibers. Neuroscience. 1987;22(2):697-707.

42. Brown A. Axonal transport of membranous and nonmembranous cargoes: a unified perspective. J Cell Biol. 2003;160(6):817-21.

43. Roy S, Zhang B, Lee VM, Trojanowski JQ. Axonal transport defects: a common theme in neurodegenerative diseases. Acta Neuropathol. 2005;109(1):5-13.

44. Hastie SB. Interactions of colchicine with tubulin. Pharmacol Ther. 1991;51(3):377-401.

45. Han Y, Malak H, Chaudhary AG, Chordia MD, Kingston DG, Bane S. Distances between the paclitaxel, colchicine, and exchangeable GTP binding sites on tubulin. Biochemistry. 1998;37(19):6636-44.

46. Takeda A, Kodama Y, Ishiwatari S, Okada S. Manganese transport in the neural circuit of rat CNS. Brain Res Bull. 1998;45(2):149-52.

47. Turner RC, Dodson SC, Rosen CL, Huber JD. The science of cerebral ischemia and the quest for neuroprotection: navigating past failure to future success. J Neurosurg. 2013;118(5):1072-85. 